\title{
BMJ Open Acupuncture treatment for knee osteoarthritis with sensitive points: protocol for a multicentre randomised controlled trial
}

\author{
Li Tang, ${ }^{1}$ Pengli Jia, ${ }^{1}$ Ling Zhao, ${ }^{2}$ Deying Kang, ${ }^{1}$ Yanan Luo, ${ }^{2}$ Jiali Liu, ${ }^{1}$ Ling Li, ${ }^{1}$ \\ Hui Zheng, ${ }^{2}$ Ying Li, ${ }^{2}$ Ning Li, ${ }^{3}$ Gordon Guyatt, ${ }^{4}$ Xin Sun ${ }^{1}$
}

To cite: Tang L, Jia P, Zhao L, et al. Acupuncture treatment for knee osteoarthritis with sensitive points: protocol for a multicentre randomised controlled trial. BMJ Open 2018;8:e23838. doi:10.1136/ bmjopen-2018-023838

- Prepublication history for this paper is available online. To view these files, please visit the journal online (http://dx.doi org/10.1136/bmjopen-2018023838).

LT and PJ contributed equally.

Received 26 April 2018

Revised 23 July 2018

Accepted 22 August 2018

Check for updates

(C) Author(s) (or their employer(s)) 2018. Re-use permitted under CC BY-NC. No commercial re-use. See rights and permissions. Published by BMJ.

${ }^{1}$ Chinese Evidence-based Medicine Center, West China Hospital, Sichuan University, Chengdu, China

${ }^{2}$ Acupuncture and Tuina School, Chengdu University of Traditional Chinese Medicine, Chengdu,

China

${ }^{3}$ Department of Integrated

Traditional Chinese and Western Medicine, West China Hospital, Sichuan University, Chengdu,

China

${ }^{4}$ Department of Health Research Methods, Evidence, and Impact (HEI), McMaster University, Hamilton, Ontario, Canada

Correspondence to

Dr Xin Sun;

sunx79@hotmail.com

\section{ABSTRACT}

Introduction There is a lack of curative medical treatment for patients with knee osteoarthritis (KOA). Acupuncture represents an important alternative therapy. According to the theory of traditional Chinese medicine and preliminary clinical evidence, the patients' acupoints and tender points may become sensitised when the body suffers from a disease state; stimulation of such sensitive points could lead to a disease improvement. It is thus hypothesised that acupuncture at highly sensitised points on patients with KOA would achieve better treatment outcomes than acupuncture at low/non-sensitised points. Previously, we conducted a pilot trial to prove the feasibility of further investigation.

Methods and analysis A three-arm, parallel, multicentre randomised controlled trial of 666 patients will be conducted at four hospitals of China. Eligible patients with KOA who consent to participate will be randomly assigned to a high-sensitisation group (patients receive acupuncture treatment at high-sensitive points), a low/non-sensitisation group (patients receive acupuncture treatment at low/ non-sensitive points) or a waiting-list group (patients receive standard acupuncture treatment after the study is concluded) via a central randomisation system using $1: 1: 1$ ratio. The primary outcome is the change of Western Ontario and McMaster Universities 0steoarthritis Index total score from baseline to 16 weeks. Outcome assessors and data analysts will be blinded and participants will be asked not to reveal their allocation to assessors. The outcome analyses will be performed both on the intention-to-treat and per-protocol population. The primary analyses will test if acupuncture at highly sensitised points would achieve statistically better treatment outcomes than acupuncture at low/non-sensitised points and no acupuncture (ie, waiting list), respectively. A small number of prespecified subgroup analyses will be conducted. Ethics and dissemination Ethics approval has been granted by the Bioethics Subcommittee of West China Hospital, Sichuan University: 2017 (Number 228). Results will be expected to be published in peer-reviewed journals. Trial registration number NCT03299439.

\section{INTRODUCTION}

Osteoarthritis, one of the most prevalent musculoskeletal diseases, is characterised
Strengths and limitations of this study

- A multicentre randomised controlled trial of 666 patients will be conducted to test if acupuncture at points with higher level of sensitisation would achieve better treatment outcomes than acupuncture at low/non-sensitisation or no acupuncture.

- The feasibility of the trial has been examined by a pilot randomised trial of 36 patients.

- This trial will be conducted using rigorous methods, such as the use of central randomisation, the blinding of data analyst and outcome assessors, and the implementation of interventions using clearly prespecified approaches.

- The loss to follow-up after randomisation in the waiting-list group might be higher than those in the two treatment groups.

by gradual loss of joint cartilage and local inflammatory processes, ${ }^{1}$ resulting in limitations in joint movement, worsening pain and reduction in quality of life. ${ }^{2}$ The knee joint is the most common site affected by osteoarthritis. ${ }^{3}$ Knee osteoarthritis (KOA) has become a major public health problem in Western societies as well as in China. In the USA, where the incidence rate was estimated nearly $0.02 \%$ among the total population, $\mathrm{KOA}$ often results in early retirement and joint replacement. ${ }^{4}$ The overall KOA prevalence in Spain, Italy, Greece and Norway was $12.2 \%, 5.4 \%, 6.0 \%$ and $7.1 \%$, respectively. ${ }^{5}$ In the Chinese population aged 60 or older, the prevalence of radiographic and symptomatic KOA was $34.1 \%$ and $11.1 \%,{ }^{6}$ similar to $33.0 \%$ and $9.5 \%$ in the US Caucasian elderly, aged 63-94 years. ${ }^{7}$

Lacking curative medical treatment for $\mathrm{KOA},{ }^{4}$ current management follows a stepwise approach that begins with conservative treatment with medications such as non-steroidal anti-inflammatory drugs (NSAIDs), 
glucosamine, topical analgesics, intra-articular injections (corticosteroids, hyaluronic acid, blood-derived products) and ends with arthroscopic knee surgery (largely discredited in randomised trials) and knee replacement. ${ }^{8}$ The age-standardised incidence rates for knee replacement surgery were estimated at 150 per 100000 personyears in Western countries. ${ }^{9}$

Given the increasing burden of knee surgery and the side effects of conventional medications, ${ }^{8}{ }^{10} 11$ patients with KOA increasingly turn to complementary and alternative treatments. ${ }^{12}$ In the UK, almost $30 \%-40 \%$ of general practices have offered complementary therapies for patients with KOA, and acupuncture is one of the most popular choices. ${ }^{13}$ Acupuncture has been used to treat osteoarthritis disorders in China since ancient times, ${ }^{14}$ and systematic reviews of randomised controlled trials (RCTs) suggested benefits of acupuncture for KOA. ${ }^{12} 1516$

As a practitioner-dependent, non-pharmacological complex intervention, ${ }^{17}$ the approach to practising acupuncture is of paramount importance. Acupuncture points represent an important issue regarding effects of acupuncture. ${ }^{18}$ According to the theory of traditional Chinese medicine (TCM), there are connections between the disease conditions and their respective points (ie, traditional acupoints and tender points (ashi points) on the surface of the human body ${ }^{19}$ ). These points become sensitised when the body suffers from a disease state. ${ }^{18}$ Stimulation of the sensitive points can lead to an improvement of disease conditions. ${ }^{18}$ Several studies have suggested that acupuncture at sensitive points might achieve higher effectiveness in different diseases when comparing against acupuncture on non-sensitive points, including myofascial pain syndrome,${ }^{20}$ lumbar disc herniation ${ }^{21}$ and pressure sores. ${ }^{22} \mathrm{~A}$ few observational studies found that certain skin points-including acupoints and tender points—of patients with KOA were sensitised. ${ }^{23-25}$ Therefore, by identifying sensitive points associated with KOA, one could hypothesise that acupuncture at more sensitised points would achieve better treatment effects on KOA.

Although a few earlier systematic reviews of RCTs addressing the effects of acupuncture for KOA concluded that acupuncture reduced pain and improved function compared with sham acupuncture, usual care or waiting list, ${ }^{12} 1516$ our systematic review of RCTs found that no existing studies specifically examined whether acupuncture at highly sensitised points versus low/non-sensitised points could achieve higher effect. ${ }^{26}$

In our effort to address the question about the effect of acupuncture at highly sensitised versus low/non-sensitised points, we have successfully conducted a pilot randomised trial of 36 patients with KOA that examined the effect of acupuncture at highly sensitised versus low/ non-sensitised points on pain. The trial showed that a definitive trial comparing acupuncture at alternative points with varying level of sensitisation was feasible, and also suggested the need to include a control group without acupuncture.
In this study, we aim to design a definitive trial to primarily examine if acupuncture at highly sensitive points, compared with low/non-sensitive points or no acupuncture (waiting list), can result in improving pain, joint function and quality of life, among patients with KOA.

\section{METHODS AND ANALYSIS}

We followed the Standard Protocol Items: Recommendations for Interventional Trials ${ }^{27}$ Clinical Trials Recommendations: Design, conduct and reporting of clinical trials for knee osteoarthritis ${ }^{28}$ and Standards for Reporting Interventions in Clinical Trials of Acupuncture $^{29}$ guidelines for the reporting of our trial protocol.

\section{Study design and setting}

A three-arm, parallel, multicentre RCT will be conducted at the Affiliated Hospital of Chengdu University of Traditional Chinese Medicine, West China Hospital of Sichuan University, the Third Affiliated Hospital of Henan University of Traditional Chinese Medicine, and Wuhan Integrated Traditional Chinese Medicine and Western Medicine Hospital, all of which are teaching and tertiary hospitals. Participants will be recruited from the outpatient departments of Acupuncture and Moxibustion, Integrated Chinese-Western Medicine and Rehabilitation Medicine. Patient enrolment started on late October 2017 and is expected to end in December 2019. Eligible and consented patients will be randomly assigned, in a ratio of 1:1:1, to receive 4-week acupuncture treatment at highly sensitive points $(\mathrm{n}=222)$, low/non-sensitive points $(\mathrm{n}=222)$ or no acupuncture treatment (ie, waiting list) $(\mathrm{n}=222)$. Participants will be assessed at baseline and 4, 8,12 and 16 weeks postintervention to determine the if acupuncture at highly sensitive points could achieve better outcomes than acupuncture at low/non-sensitive points or no acupuncture treatment. The design of the trial is summarised in figure 1.

\section{Participants}

Participants aged 40 years or older and diagnosed with mild or moderate KOA are eligible to participate in the study. The following criteria are used for diagnosis of KOA according to the Chinese Guideline for the Medical Management of Osteoarthritis ${ }^{30}$ : (1) refractory knee pain for most days in the last month; (2) joint space narrowing, sclerosis or cystic change in subchondral bone (as demonstrated by X-ray); (3) laboratory examinations of arthritis: clear and viscous synovial fluid ( $\geq 2$ times) and white cell count $<2 \times 10^{\wedge} 9 / \mathrm{L}$; (4) aged 40 years or older; (5) morning stiffness continues less than $30 \mathrm{~min}$; (6) bone sound exists when joints was taking flexion and/or extension. If a patient meets the following combination of criteria ( 1 and 2), (1, 3, 5 and 6$)$ or (1, 4, 5 and 6$)$, a diagnosis of KOA is confirmed.

Patients will be excluded if they meet any of the following: (1) diagnosed with conditions leading to 


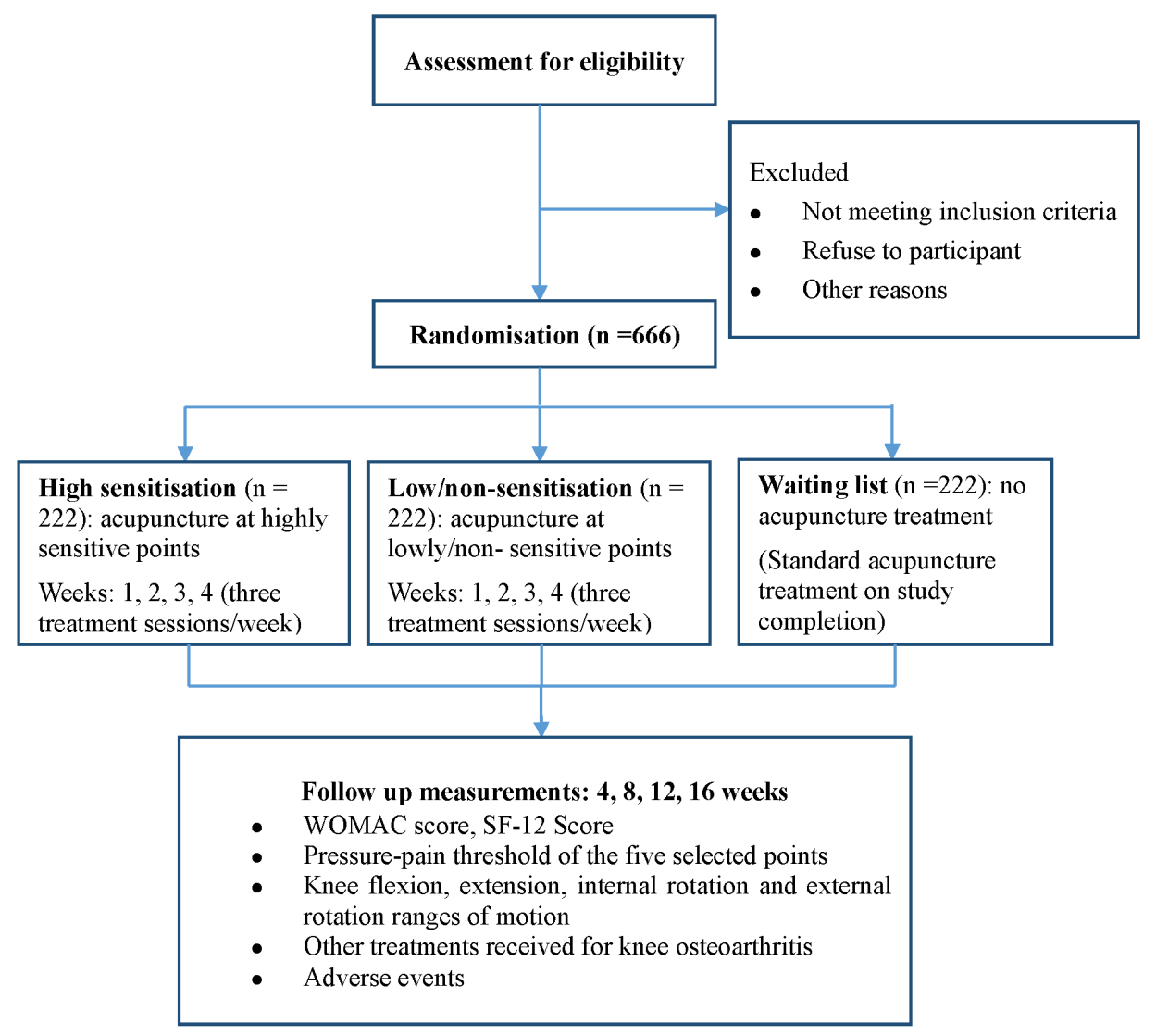

Figure 1 Trial design of the acupuncture treatment for knee osteoarthritis. SF-12, 12-item Short Form Health Survey; WOMAC, Western Ontario and McMaster Universities Osteoarthritis Index.

skeletal disorders, such as tuberculosis, tumours or rheumatism of the knee joint and rheumatoid arthritis; (2) present with sprain or trauma in the lower limb; (3) unable to walk properly due to foot deformity or pain; (4) cannot answer the questionnaire due to mental disorders and/ or intellectual disability; (5) present with comorbidities including severe cardiovascular disease, liver or kidney impairment, immunodeficiency, diabetes mellitus, blood disorder or skin disease; (6) females who are pregnant or lactating; (7) are using or have used physiotherapy treatments for osteoarthritis knee pain in the past month; (8) have used intra-articular injection of glucocorticoid or viscosupplementation in the past 6 months; (9) received knee replacement surgery on the affected side(s); (10) diagnosed with severe (stage 4, according to Kellgren and Lawrence radiographic classification) or late clinical stage of KOA; (11) have a swollen knee or positive result of floating patella test or (12) are participating in the other clinical trials of acupuncture.

Potentially eligible patients will be screened by the research physicians and research staff through physical examination and clinical tests. Patients will be informed of the aims and nature of the research both verbally and via an information sheet. They will be requested to complete and sign a consent form before enrolment. Patients information will remain confidential and they will have the right to withdraw without prejudice at any time.

\section{Randomisation}

Eligible patients who consent to participate will be randomly assigned to a high-sensitisation group (patients receive acupuncture treatment at high-sensitive points), a low/non-sensitisation group (patients receive acupuncture treatment at low/non-sensitive points) or a waiting-list group (patients receive standard acupuncture treatment after the study is concluded) via a central randomisation system for clinical research using 1:1:1 ratio. Randomisation will be stratified by participating site in block sizes of 3 or 6 . The study coordinator at each site will ensure that the informed consent form has been obtained from each participant prior to randomisation. He/she will then $\log$ onto the central randomisation system using a password-protected account and enter inclusion and exclusion criteria to ensure eligibility before entering the patient's name, identification card number to generate a random sequence.

\section{Identification, measurement and selection of acupoints and tender points for acupuncture}

Patients randomly allocated to either acupuncture at highly sensitive points (high-sensitisation group) or acupuncture at low/non-sensitive points (low/non-sensitisation group) will first be measured for sensitisation intensity of acupoints or tender points. Those points will then be ranked according to the intensity of sensitisation 

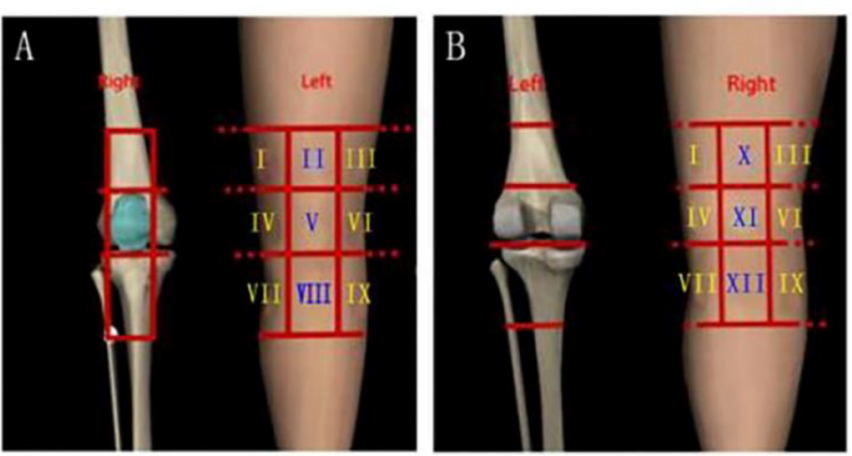

Figure 2 The front and back testing zones of the knee region.

(figure 1). Thereafter, patients randomly allocated to high-sensitisation group will receive acupuncture at the five points with highest sensitisation, and those allocated to low/non-sensitisation group will receive acupuncture at the five points with lowest sensitisation. Patients randomly allocated to the waiting-list group (no acupuncture group) will not be measured for sensitisation intensity.

There are several types of point sensitisation, such as pain, force and heat. ${ }^{183132}$ Pain sensitisation, which can be quantitatively measured, will be adopted in this trial, since KOA is mainly characterised with pain and limitations in joint movement. The pressure-pain threshold, measured by electronic von Frey detector (2390 series, IITC Life Science), will be used by trained acupuncturists to quantify the intensity of sensitisation in participants from the high and low/non-sensitisation groups.

\section{Identification of candidate acupoints and tender points for sensitisation measurement}

Based on literature ${ }^{33}$ and expert consensus, we identified 13 candidate acupoints for treating KOA, namely Heding (EX-LE2), Neixiyan (EX-LE4), Dubi (ST35), Xuehai (SP10), Liangqiu (ST34), Yinlingquan (SP9), Yanglingquan GB34), Zusanli (ST36), Weizhong (BL40), Yingu (KI10), Xiguan (LR7), Ququan (LR8), Weiyang (BL39).

We will also identify candidate tender points using prespecified approach. First, the front and back of the knee regions of each participant are divided into a total of 12 testing zones (figure 2). The surface marking methods are as follows: (1) draw a line horizontally three body inches (cun) above the patella (the upper boundary of the testing zones); (2) draw a line horizontally across Zusanli (ST36) (the lower boundary of the testing zones); (3) draw two lines which horizontally trisect the area between the upper and lower boundaries; (4) in the front region, draw two lines vertically across the top corners of the basis patellae respectively; (5) in the back region, draw two lines which vertically trisect the popliteal area. Tender points in the testing areas will be identified when the participant reports the sensation of pain, soreness, distention or sometimes comfort while acupuncturist pressing vertically downward about $6-10 \mathrm{~mm}$ at an even speed with the thumb.

Measurements of acupoints and tender points for pressure-pain threshold

The electronic von Frey detector (2390 series, IITC Life Science) will be used by trained acupuncturists to measure the pressure-pain thresholds of selected acupoints and tender points. The acupuncturist moves the probe tip vertically downward the skin at an even speed. He/she will remove the probe immediately once the patient feels the pain. The data revealed by the detector (reported as numbers) will then be recorded as the pressure-pain threshold. Each point will be tested two times at an interval of $2 \mathrm{~min}$. If the difference of two data values is larger than 15, the point will be measured for the third time. The average of the two values with the smallest difference will be taken as the final threshold.

Selection of highly and lowly/non-sensitive points for acupuncture All of the 13 candidate acupoints or identified tender points will be ranked based on their pressure-pain threshold. The five points with the lowest pain threshold are identified as the highly sensitive points, whereas the five points with the highest pain threshold are selected as the lowly/non-sensitive points.

\section{Interventions}

Participants in the high-sensitisation group receive acupuncture treatment at the five highly sensitive points. The acupuncturists were specialists in TCM and received specialised training in the acupuncture protocols prior to the start of the study. Sterile, single-use filiform acupuncture needles (Hwato Needles, Sino-foreign Joint Venture Suzhou Hwato Medical Instruments, China) with a length of $40 \mathrm{~mm}$ and a diameter of $0.30 \mathrm{~mm}$ will be inserted to a depth of $15-30 \mathrm{~mm}$ in acupoints. The insertion will be followed by stimulation performed with lifting and thrusting combined with twirling and rotating the needles sheath in order to produce the sensation known as achieve 'Deqi' (refers to a sensation of soreness, numbness, distention or radiating considered to be indicative of effective needling). ${ }^{35}$ The needles placed in the acupoints will be manually stimulated every $15 \mathrm{~min}$ and removed after $30 \mathrm{~min}$. Participants receive three treatment sessions per week (every other day) for four consecutive weeks. Participants in the low/non-sensitisation group receive acupuncture treatment at the five low/non-sensitive points. All other treatment settings will be the same as in the high-sensitisation group. Patients in the waiting-list group will not receive any acupuncture intervention during the study. However, for the ethnic consideration, we will offer free, non-study standard acupuncture treatment at the following traditional acupoints after the study is completed: DUBI (ST35), NEIXIYAN (EX-LE4), YANGLINGQUAN GB34), ZUSANLI (ST36) and XUEHAI (SP10). 
In the high and low/non-sensitisation groups, the intervention will be performed on the side of the affected joint for patients with unilateral KOA, whereas patients with bilateral KOA will have their most painful knee assessed and intervened. Furthermore, the non-trial affected lower limbs will receive standard acupuncture treatment on DUBI (ST35), NEIXIYAN (EX-LE4), YANGLINGQUAN GB34), ZUSANLI (ST36) and XUEHAI (SP10).

All the participants will be advised not to take any other treatments for KOA. However, NSAIDs are allowed if patients have intolerable pain and the outcome assessment is not scheduled in the next 48 hours. In addition, non-acupuncture treatments, such as application of medicinal liquor on the knee, heat therapy using the Teding Diancibo $\mathrm{Pu}$ device, massage and moxibustion, are allowed if patients in the waiting-list group, but not the two acupuncture groups, request treatment during the study period. Patients will be asked to document all such treatments (including the name, dosage/frequency and duration of treatment) receiving for KOA.

\section{Follow-up and outcomes}

The pain, stiffness and physical function of the knee joint, quality of life and knee ranges of motion (ROMs) of all the participants, as well as pressure-pain threshold of the selected acupuncture points and the safety of treatment of patients in the high and low/non-sensitisation groups will be measured at follow ups at 4, 8, 12 and 16 weeks after randomisation.

The following measures will be taken to optimise the retention of participants: free acupuncture treatments for other diseases provided they do not affect the treatment of KOA; text messages and phone calls to remind the participants about the impending appointments; flexibility in making appointment to suit the participants' schedule; in addition to contact details of the participants, phone numbers of close family members will also be recorded on permission to facilitate tracking.

The outcome assessments will be performed by nurses or completed by patients in a separate space at the outpatient department of each participating site. All of the outcome assessors will be trained on conducting interviews and performing measurements before the study begins and will follow a standard protocol. The schedule of measurements is present in table 1 .

\section{Primary outcome}

The primary outcome is the change of Western Ontario and McMaster Universities Osteoarthritis Index (WOMAC) total score from baseline to 16 weeks. The

Table 1 Measurements to be taken at each point in trial

\begin{tabular}{|c|c|c|c|c|c|c|}
\hline \multirow[b]{3}{*}{ Timepoint } & \multicolumn{6}{|l|}{ Study period } \\
\hline & \multirow{2}{*}{$\begin{array}{l}\text { Enrolment } \\
-1 \text { week } \\
\text { (-7 to } 0 \text { day) }\end{array}$} & \multirow{2}{*}{$\begin{array}{l}\text { Allocation } \\
\text { Day } 0\end{array}$} & \multicolumn{3}{|c|}{ Postallocation } & \multirow{2}{*}{$\begin{array}{l}\text { Postallocation } \\
16 \text { weeks } \\
\text { ( } \pm 3 \text { days) }\end{array}$} \\
\hline & & & $\begin{array}{l}4 \text { weeks } \\
( \pm 3 \text { days })\end{array}$ & $\begin{array}{l}8 \text { weeks } \\
( \pm 3 \text { days) }\end{array}$ & $\begin{array}{l}12 \text { weeks }( \pm 3 \\
\text { days) }\end{array}$ & \\
\hline \multicolumn{7}{|l|}{ Enrolment } \\
\hline Eligibility screen & $x$ & & & & & \\
\hline Informed consent & $x$ & & & & & \\
\hline Allocation & & $x$ & & & & \\
\hline \multicolumn{7}{|l|}{ Interventions } \\
\hline High-sensitisation group & & & $\mathrm{x}$ & & & \\
\hline Low/non-sensitisation group & & & $x$ & & & \\
\hline Waiting-list group & & & $\mathrm{x}$ & & & \\
\hline \multicolumn{7}{|l|}{ Assessments } \\
\hline X-ray examination of the knee joint & $x$ & & & & & \\
\hline Measurement of sensitisation & $x$ & & & & & \\
\hline Intensity & & & $x$ & $x$ & $x$ & $x$ \\
\hline Measurement of pressure-pain & $x$ & & $\mathrm{x}$ & $x$ & $\mathrm{x}$ & $\mathrm{x}$ \\
\hline Threshold of the five selected points & $x$ & & $\mathrm{x}$ & $x$ & $\mathrm{x}$ & $\mathrm{x}$ \\
\hline WOMAC score & $x$ & & $\mathrm{x}$ & $x$ & $\mathrm{x}$ & $x$ \\
\hline SF-12 score & & & $\mathrm{x}$ & $x$ & $\mathrm{x}$ & $\mathrm{x}$ \\
\hline Knee ranges of motion & $x$ & & $\mathrm{x}$ & $x$ & $\mathrm{x}$ & $\mathrm{x}$ \\
\hline \multicolumn{7}{|l|}{ Adverse events } \\
\hline $\begin{array}{l}\text { Other treatments received for knee } \\
\text { osteoarthritis }\end{array}$ & & & & & & \\
\hline
\end{tabular}

SF-12, 12-item Short Form Health Survey; WOMAC, Western Ontario and McMaster Universities Osteoarthritis Index; X, measurements to be taken at this point. 
WOMAC is a valid and reliable instrument that has been used widely in a variety of clinical trials involving patients with KOA. It has been translated and validated in different languages including Chinese. ${ }^{36}$ The Chinese version of WOMAC consists of 24 items assessing the patients with KOA pain ( 5 items), stiffness ( 2 items) and physical function (17 items). Each of the 24 items will be graded on a Visual Analogue Scale (VAS) ranging from 0 to 10, with higher scores reflecting more pain, stiffness and poorer physical function. The score ranges for the pain, stiffness and physical function subscales are, respectively, 0-50, $0-20$ and $0-170$, resulting in a total range of $0-240$.

\section{Secondary outcomes}

The secondary study outcomes are: (1) the change of 12-item Short Form Health Survey (SF-12) score from baseline to 16 weeks, (2) the changes of knee ROMs from baseline to 4 and 16 weeks and (3) the changes of pressure-pain threshold of the five selected acupuncture points from baseline to 4 and 16 weeks.

The SF-12 is a shorter version of the SF-36 and has been used to measure health-related quality of life for patients with KOA. ${ }^{37}$ It includes 12 items: 2 items on physical functioning, 2 items on role limitations because of physical health problems, 1 item on bodily pain, 1 item on general health perceptions, 1 item on vitality (energy/fatigue), 1 item on social functioning, 2 items on role limitations because of emotional problems and 2 items on general mental health (psychological distress and psychological well-being). The validated Chinese version of SF-12 will be used in the present trial. ${ }^{38}$ The knee ROMs will be assessed both actively and passively by using a standard goniometer. It will include flexion, extension, internal rotation and external rotation. The pressure-pain threshold of the five selected acupuncture points will be measured using the electronic von Frey detector.

\section{Assessment of safety}

All unfavourable or unintended events affecting patients in the study, such as bleeding, bruising and/or soreness at the needle sites (assessment taken immediately after acupuncture by acupuncturists' and patients' report) and elevated blood pressure (standard measurement at each follow-up), will be recorded using prespecified questionnaire and followed up for the duration of the study or until resolution.

\section{Blinding}

Study investigators, acupuncturists and participants will be aware of the treatment allocation. Outcome assessors and data analysts will be blinded and participants will be asked not to reveal their allocation to assessors. In addition, we will have blinded interpretation of the study results to minimise misleading data interpretation. ${ }^{39}$

\section{Sample size}

Our primary study hypothesis is that acupuncture on high-sensitive points (ie, experimental group) would achieve more reduction in the total WOMAC score than acupuncture on low/non-sensitive points (ie, active control group) or waiting-list group (ie, no treatment group).

The sample size estimation was based on the mean difference in the change of total WOMAC score from baseline given the estimates obtained from our pilot trial. The following assumptions were made to calculate the sample size: a mean difference of 12 between the high and low/non-sensitisation groups, SD of total score of 33, a two-sided significance level of 0.025 (adjusted for multiple testing) and a power of 0.9 . With these assumptions, a sample size 189 patients per arm is required to provide a power of $90 \%$ at the alpha level of 0.025 to detect a difference of 12 points between the high and low/non-sensitisation groups. This sample size would provide adequate power to detect the difference between high-sensitisation group versus waiting-list group, on the ground that the treatment effect between high-sensitisation group and low/non-sensitisation group would be smaller than that between high-sensitisation group and waiting-list group.

To allow for a loss to follow-up of $10 \%$, a minimum sample size of 666 patients (222 patients per arm) at baseline was required.

\section{Monitoring}

We will develop an independent data monitoring committee (DMC), responsible for the monitoring for quality and regulatory compliance of the trial, as well as ensuring the safety of participating patients. The DMC will consist of five members with expertise in acupuncture, KOA management, trial methodology and biostatistics. The DMC meeting will be held once a year; at the request of DMC, the meeting may take place every 6 months. We will develop a procedural document for the DMC meeting, and strictly follow the document.

\section{Statistical analysis}

We will descriptively summarise patient characteristics, medical characteristics, outcome variables and the adverse events. Group comparisons will be undertaken using $\chi^{2}$ tests for categorical characteristics, and either analysis of variance or Mann-Whitney $\mathrm{U}$ tests for continuous variables.

The primary analyses will examine if acupuncture at highly sensitive points will achieve statistically better treatment outcomes than acupuncture at low/non-sensitisation points and waiting-list group, respectively. To accommodate the correlation of repeated measures from the same participant and the nesting of observations within research sites, generalised linear models with random effects (participants and research sites) will be fitted to assess the intervention effect on outcome variables over time, while accounting for the effects of potential confounders (eg, age, gender, body mass index (BMI) and other treatments). We will also examine, in an exploratory analysis, if acupuncture at low/ non-sensitisation would achieve statistically better outcomes than waiting list.

The outcome analyses will be performed both on the intention-to-treat population, which includes all patients 
randomised, and on the per-protocol population, which includes eligible patients who adhere to the planned treatment and follow ups. We will use the last value carried forward method to impute missing data for the primary and secondary outcomes.

We will also conduct subgroup analyses according to the following prespecified baseline variables: BMI $(<18.5$ vs $\left.18.5-23.9 \mathrm{vs} 24 \mathrm{~kg} / \mathrm{m}^{2}\right),{ }^{40}$ severity of pain measured by VAS (absent or minor 1-3 vs moderate 4-6 vs severe 7-10), type of KOA (single vs bilateral KOA), stage of KOA according to $\mathrm{X}$-ray imaging ${ }^{41}$ and duration of disease.

\section{Study committees}

We have developed the following committees for the trial. Study principal investigator: Xin Sun. Steering committee: Xin Sun (chair), Ying Li, Fanrong Liang, Gordon Guyatt. Methods Center staff: Xin Sun, Li Tang, Jiali Liu, Pengli Jia, Ling Li and Zhibin Liu. Data monitoring committee: Chen Yao (chair), Zhaoxiang Bian, Hongcai Shang and Honglai Zhang. Site audit committee: Jiali Liu, Li Tang and Zhibin Liu.

\section{Patient and public involvement}

The patients and public were not involved in planning and design of this study.

\section{DISCUSSION}

KOA is a major health concern worldwide and its disease burden continues to increase due to population ageing. Standard treatments for KOA, such as NSAIDs glucosamine and intra-articular injections, are often not effective. In the face of the limitations of alternative treatments, acupuncture has long been an alternative in China and has received increasing attention in many Western countries. ${ }^{42}$ A number of RCTs have been conducted to test the effectiveness of acupuncture on KOA. Based on published systematic reviews and meta-analysis of these RCTs, acupuncture seems to be a promising treatment for KOA. ${ }^{12}$

However, very few trials with serious limitations have specifically examined if acupuncture at points with varying sensitisation would achieve different treatment outcomes. According to the theory of TCM, a sensitised point may reflect the pathological phenomenon of disease on the surface of the human body and represent an optimal target for acupuncture treatment. ${ }^{18}$ The theory that acupuncture at sensitive points leads to a better therapeutic effect has been widely discussed, but limited research has been conducted to address the issue among patients with KOA. If this hypothesis was proved, the findings would have profound impact on the theory and practice of acupuncture on KOA. First, the trial would contribute to a better understanding of the effectiveness of acupuncture for KOA. Second, it would provide compelling evidence on the selection of acupuncture points to achieve improved KOA outcomes.
Third, the resulting findings may change the guideline for practising acupuncture on KOA.

We have carefully designed the present RCT and include thorough details about plan for implementation, aiming to offer trustworthy evidence for the effects of acupuncture for KOA. Specifically, we will examine beneficial effects of stimulation of sensitised points on pain reduction, and improvement of joint function and quality of life. We will use computer-generated randomisation and central randomised allocation concealment to minimise selection bias, and apply blocked randomisation and stratification of randomisation by site to ensure prognostic balance between intervention groups. Those supervising or administering outcome assessors will be blinded to the group allocation. Also, standardised and validated questionnaires will be administered to ensure credible measurement of patient health status.

Our study also has a few limitations. First, we did not include the shame procedure as an active control. Thus, the placebo effect may not be well parcelled out. However, our primary objective is to identify whether there is difference between in the acupuncture between highly sensitive and low/non-sensitive points. Thus, the low/non-sensitive points would serve as the primary active control. The inclusion of waiting list will also be used a non-treatment control. Second, we included participants with any level of pain. However, the inclusion of patients with VAS less than 4 may be a limitation, the level of pain symptoms at baseline should be of sufficient severity to allow for detection of change. Third, we did not include a baseline variable for stratification at randomisation, which might affect the baseline of important prognostic factors. Another possible limitation of this RCT is a potentially higher rate of loss to follow-up after randomisation in the waiting-list group than those in the two treatment groups. However, several measures will be taken throughout the study to maximise the participants' retention.

In summary, we will conduct a definitive multicentre RCT to test if acupuncture at highly sensitised points would achieve better treatment outcomes than acupuncture at low/non-sensitised points or no acupuncture treatment in 666 patients diagnosed with KOA. We will use rigorous methods to minimise bias and set up several working committees to ensure quality conduct of the trial. The resulting findings would have important impact on the practice of acupuncture for patients with KOA.

\section{ETHICS AND DISSEMINATION}

The case report forms (CRFs) will be stored in a locked cabinet at the participating hospitals and accessible by the research team only. On completion of the trial and data checking, the CRFs will be transferred to be securely archived at SiSichuanniversity for 10 years before being destroyed. The trial database will be anonymised, password protected and securely held. Patient identifiable data are shared only within the clinical team on a need-to-know basis to provide clinical care and ensure appropriate follow-up. 
The aggregated research findings will be presented at national and international scientific conferences and be submitted for publication in peer-reviewed journals.

Contributors XS conceived the study and acquired the funding. XS, GG, LT, PJ, LZ, $\mathrm{DK}, \mathrm{YL}, \mathrm{JL}, \mathrm{LL}, \mathrm{HZ}, \mathrm{YL}$ and NL participated in improving the study design. $\mathrm{LT}$ and $\mathrm{PJ}$ drafted the manuscript. XS, GG, LT, PJ, LZ, DK, YL, JL, LL, HZ, YL and NL critically revised the manuscript. All authors read and approved the final manuscript. XS is the guarantor

Funding This study is funded by the National Natural Science Foundation of China (grant number 81590955), and XS is supported by the 'Thousand Youth Talents Plan' of China (grant number D1024002).

Competing interests None declared.

Patient consent Obtained.

Ethics approval Ethics approval has been granted by the Bioethics Subcommittee of West China Hospital, Sichuan University: 2017 (Number 228).

Provenance and peer review Not commissioned; externally peer reviewed.

Open access This is an open access article distributed in accordance with the Creative Commons Attribution Non Commercial (CC BY-NC 4.0) license, which permits others to distribute, remix, adapt, build upon this work non-commercially, and license their derivative works on different terms, provided the original work is properly cited, appropriate credit is given, any changes made indicated, and the use is non-commercial. See: http://creativecommons.org/licenses/by-nc/4.0/.

\section{REFERENCES}

1. Ezzo J, Hadhazy V, Birch S, et al. Acupuncture for osteoarthritis of the knee: a systematic review. Arthritis Rheum 2001;44:819-25.

2. Manyanga T, Froese $M$, Zarychanski R, et al. Pain management with acupuncture in osteoarthritis: a systematic review and meta-analysis. BMC Complement Altern Med 2014;14:312.

3. Chen R, Chen M, Kang M, et al. The design and protocol of heat-sensitive moxibustion for knee osteoarthritis: a multicenter randomized controlled trial on the rules of selecting moxibustion location. BMC Complement Altern Med 2010;10:32.

4. Oliveria SA, Felson DT, Reed JI, et al. Incidence of symptomatic hand, hip, and knee osteoarthritis among patients in a health maintenance organization. Arthritis Rheum 1995;38:1134-41.

5. Guillemin F, Rat AC, Mazieres B, et al. Prevalence of symptomatic hip and knee osteoarthritis: a two-phase population-based survey. Osteoarthritis Cartilage 2011;19:1314-22.

6. Zhang Y, Xu L, Nevitt MC, et al. Comparison of the prevalence of knee osteoarthritis between the elderly Chinese population in Beijing and whites in the United States: the Beijing osteoarthritis study. Arthritis Rheum 2001;44:2065-71.

7. Felson DT, Naimark A, Anderson J, et al. The prevalence of knee osteoarthritis in the elderly. The framingham osteoarthritis study. Arthritis Rheum 1987;30:914-8.

8. Soni A, Joshi A, Mudge N, et al. Supervised exercise plus acupuncture for moderate to severe knee osteoarthritis: a small randomised controlled trial. Acupunct Med 2012;30:176-81.

9. Clausen B, Holsgaard-Larsen A, Søndergaard J, et al. The effect on knee-joint load of instruction in analgesic use compared with neuromuscular exercise in patients with knee osteoarthritis: study protocol for a randomized, single-blind, controlled trial (the EXERPHARMA trial). Trials 2014;15:444.

10. Blower AL, Brooks A, Fenn GC, et al. Emergency admissions for upper gastrointestinal disease and their relation to NSAID use. Aliment Pharmacol Ther 1997:11:283-91.

11. McGettigan P, Henry D. Cardiovascular risk and inhibition of cyclooxygenase: a systematic review of the observational studies of selective and nonselective inhibitors of cyclooxygenase 2. JAMA 2006;296:1633-44.

12. Cao L, Zhang XL, Gao YS, et al. Needle acupuncture for osteoarthritis of the knee. A systematic review and updated metaanalysis. Saudi Med J 2012;33:526-32.

13. Thomas KJ, Nicholl JP, Fall M. Access to complementary medicine via general practice. Br J Gen Pract 2001;51:25-30.

14. Magden ER, Haller RL, Thiele EJ, et al. Acupuncture as an adjunct therapy for osteoarthritis in chimpanzees (Pan troglodytes). J Am Assoc Lab Anim Sci 2013;52:475-80.
15. Adrian WNF, Mike C, Panos B. The effectiveness of acupuncture for osteoarthritis of the knee - a systematic review. Acupunct Med 2006:40-8.

16. Manheimer E, Linde K, Lao L, et al. Meta-analysis: acupuncture for osteoarthritis of the knee. Ann Intern Med 2007;146:868-77.

17. Kim EJ, Lim CY, Lee EY, et al. Comparing the effects of individualized, standard, sham and no acupuncture in the treatment of knee osteoarthritis: a multicenter randomized controlled trial. Trials 2013;14:129.

18. Chen RX, Kang MF, Chen MR. [Return of Qibo: on hypothesis of sensitization state of acupoints]. Zhongguo Zhen Jiu 2011;31:134-8.

19. Zhu B. [The plasticity of acupoint]. Zhongguo Zhen Jiu 2015;35:1203-8.

20. Chen RX, Kang MF, He WL, et al. [Moxibustion on heat-sensitive acupoints for treatment of myofascial pain syndrome: a multi-central randomized controlled trial]. Zhongguo Zhen Jiu 2008;28:395-8.

21. Tang FY, Huang CJ, Chen RX, et al. [Observation on therapeutic effect of moxibustion on temperature-sensitive points for lumbar disc herniation]. Zhongguo Zhen Jiu 2009;29:382-4.

22. Zhang $\mathrm{C}$, Xiao H, Chen R. Observation on curative effect of moxibusting on heat-sensitive points on pressure sores. Chinese $J$ Tradit Chin Med 2010;25:478-9.

23. Chen R, Kang M. Acupoint heat-sensitization and its clinical significance. J Tradit Chin Med 2006;47:905-6.

24. Duan Q, Yuan F, Liang A, et al. Clinical observation of heat sensitive moxibustion on acupoints in 120 cases of knee osteoarthritis. New Chin Med 2014;46:171-3.

25. Xie H, Chen R, Xu F, et al. Comparative study of heat-sensitive moxibustion in the treatment of knee osteoarthritis. Chin Acup Moxibustion 2012;32:223-32.

26. Jia P, Tang L, Yu J, et al. Risk of bias and methodological issues in randomised controlled trials of acupuncture for knee osteoarthritis: a cross-sectional study. BMJ Open 2018;8:e019847.

27. Chan AW, Tetzlaff JM, Altman DG, et al. SPIRIT 2013 statement: defining standard protocol items for clinical trials. Ann Intern Med 2013;158:200-7.

28. McAlindon TE, Driban JB, Henrotin Y, et al. OARSI Clinical trials recommendations: design, conduct, and reporting of clinical trials for knee osteoarthritis. Osteoarthritis Cartilage 2015;23:747-60.

29. MacPherson H, Altman DG, Hammerschlag R, et al. Revised STandards for Reporting Interventions in Clinical Trials of Acupuncture (STRICTA): extending the CONSORT statement. PLOS Med 2010;7:e1000261.

30. Chinese Orthopaedic Association. Guidelines for the medical management of osteoarthritis (Year 2007). Ortho J Chin 2014;22:287-8.

31. Ben H, Li L, Rong P-J, et al. Observation of pain-sensitive points along the meridians in patients with gastric ulcer or gastritis. Evid Based Complementary Altern Med 2012;2012:1-7.

32. He S, Fu Y, Shi N. Clinical observation of the therapeutic effect on cervical spondylosis treated by force-sensitive points manipulation. Pract Clin J Integrated Trad Chin Western Med 2014;14:1-2.

33. Li H, Ma Z, Fang Z, et al. The rule of acupoints' selection in acupuncture and moxibustion for knee osteoarthritis in clinic using data mining analysis. World Sci Tech/Modernization Trad Chin Med Materia Medica 2016;18:1233-40.

34. Li D. Literature research on acupoint selection rules and moxibustion methods in treatment of knee degenerative osteoarthritis by warming acupuncture. For all Health 2015;9:326-7.

35. Yuan HW, Ma LX, Zhang P, et al. An exploratory survey of deqi sensation from the views and experiences of chinese patients and acupuncturists. Evid Based Complement Alternat Med 2013;2013:1-8.

36. Xie F, Li SC, Goeree R, et al. Validation of Chinese Western Ontario and McMaster Universities Osteoarthritis Index (WOMAC) in patients scheduled for total knee replacement. Qual Life Res 2008;17:595-601.

37. Fang WH, Huang GS, Chang HF, et al. Gender differences between WOMAC index scores, health-related quality of life and physical performance in an elderly Taiwanese population with knee osteoarthritis. BMJ Open 2015;5:e008542.

38. Lam CL, Tse EY, Gandek B. Is the standard SF-12 health survey valid and equivalent for a Chinese population? Qual Life Res 2005;14:539-47.

39. Järvinen TL, Sihvonen R, Bhandari M, et al. Blinded interpretation of study results can feasibly and effectively diminish interpretation bias. $J$ Clin Epidemiol 2014;67:769-72.

40. Chen C, Lu FC. Department of Disease Control Ministry of Health, PR China. The guidelines for prevention and control of overweight and obesity in Chinese adults. Biomed Environ Sci 2004;17 Suppl:1-36.

41. SCRIBD. Kellgren Lawrence Grading Scale of the osteoarthritis of the knee. https://www.scribd.com/./106388906/Kellgren-LawrenceGrading-Scale (accessed 25 Apr 2018).

42. Vanderploeg K, Yi X. Acupuncture in modern society. J Acupunct Meridian Stud 2009;2:26-33. 